\title{
L'exploitation hydrothermique des nappes par pompe à chaleur eau-eau
}

\author{
Hydrothermal exploitation of Groundwater \\ by water-water heat pump
}

\author{
J.P. Sauty et J.Y. Ausseur
}

Bureau de recherches géologiques et minières

Service géologique national, Orléans

Dans l'utilisation thermique des eaux souterraines pour le chauffage ou le refroidissement par pompe à chaleur, la réinjection des eaux par un second forage préserve lo ressource en eau, mais perturbe les températures du milieu géologique, d'où une incidence pour l'installation elle-même (recyclage) et pour d'autres utilisateurs potentiels en aval du puits de rejet. Après un examen rapide de l'évolution de cette nouvelle demande en eaux souterraines et de l'état actuel de la réglementation française, on s'attache à définir les moyens d'évaluation de l'impact de ces installations sur les réserves. Enfin, la possibilité de faciliter la gestion des eaux souterraines exploitées à des fins diverses, grâce à la mise en auvre de modèles de gestion hydrothermique, est abordée.
When groundwater is used for heating or cooling by means of a heat pump, reinjection of the waters by a second drill hole preserves the water resource but disturbs the temperatures of the geological medium, which has consequences not only on the facility itself (recirculation) but also on other potential users downstream of the reject well. After a rapid review of the trend in this new demand for groundwater and the present state of French regulations, the paper attempts to define means to evaluate the impact of these facilities on the reserves. Finally, the possibility of facilitating management of groundwater used for various purposes by means of hydrothermal management models, is approached.

\section{Introduction}

\section{Rappel historique}

L'exploitation thermique des eaux souterraines par pompe à chaleur se pratique depuis plusieurs décennies avec le refroidissement d'installations industrielles ou la climatisation d'immeubles. Parfois, on utilisait également en période froide, la chaleur dégagée par les condenseurs des groupes frigorifiques gagnant ainsi sur les deux tableaux (BERNIER, 1979). L'intérêt pour les pompes à chaleur en tant que moyen de chauffage, ne s'est véritablement manifesté que lorsque des menaces sérieuses ont pesé sur les approvisionnements énergétiques : 35 unités ont été installées en Suisse durant la seconde guerre mondiale (DumiNIL, 1979). L'essor contemporain de ce matériel voit ses origines dans les crises pétrolières successives depuis 1973. De fait, les difficultés technologiques rencontrées par les constructeurs ont retardé jusqu'à la fin des années
70 la production en série d'un matériel fiable (BERNIER, ib). Depuis, le nombre de pompes à chaleur exploitées en France a bénéficié d'un accroissement quasi exponentiel (fig. 1); il a pratiquement triplé en 2 ans (entre 1980 et 1982).

Rappel sur le fonctionnement des pompes à chaleur (fig. 2)

Nous rappellerons pour mémoire le principe du fonctionnement de la pompe à chaleur (PAC en abrégé). Il repose sur la circulation d'un fluide frigorigène en boucle fermée reliant deux échangeurs, chacun au contact d'une source de chaleur à température différente $\left(T_{1}\right.$ et $\left.T_{2}<T_{1}\right)$. Un compresseur et un détendeur font varier les pressions sur la boucle. Le fluide et les pressions du cycle thermodyna- 


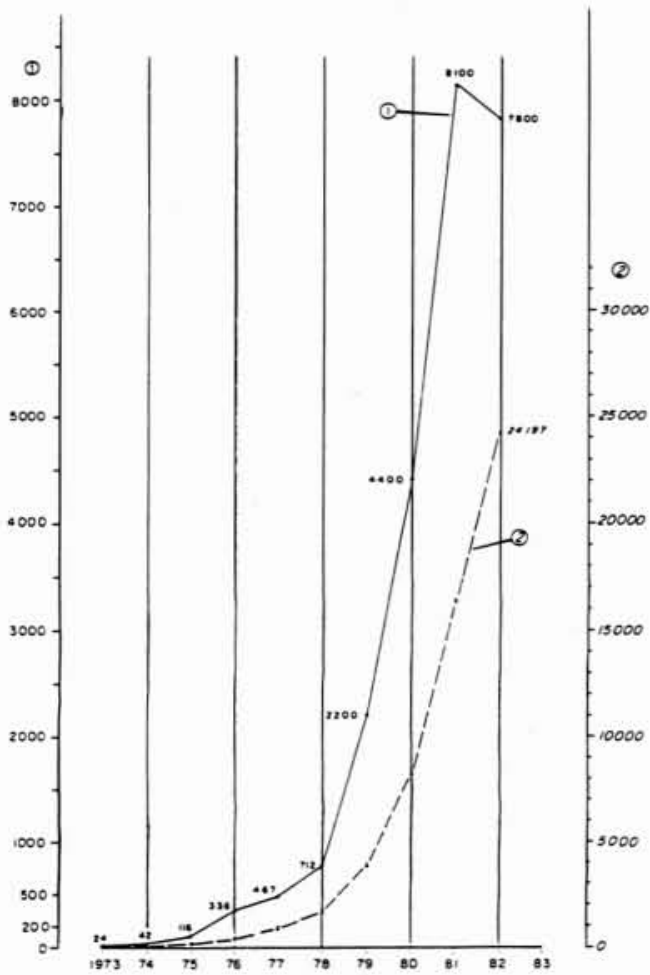

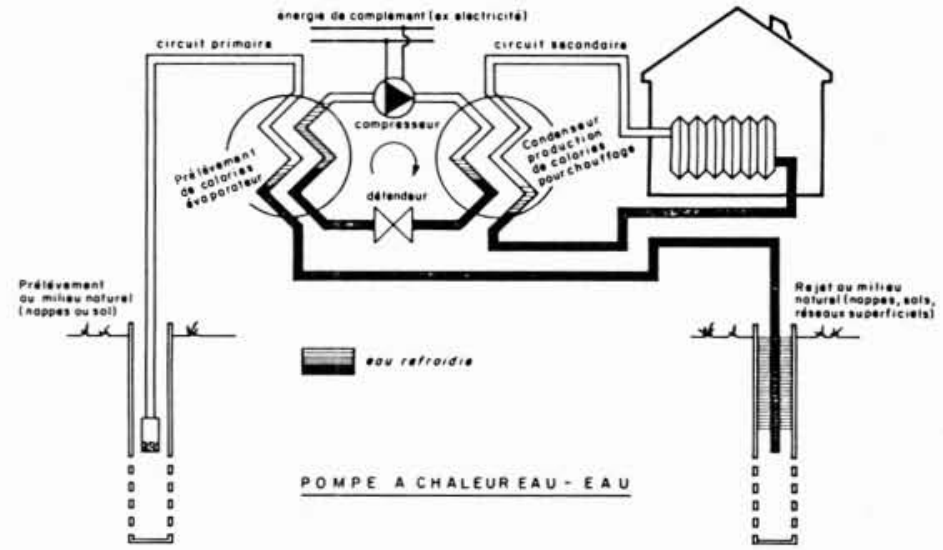

Figure 2. - Principe de la pompe à chaleur eau-eau sur doublet de forages.

Figure 1. - Nombres de pompes à chaleur eau/eau installées en France (toutes puissances groupées) annuels (1) - échelle de gauche - et cumulés (2) -échelle de droite- de 1973 à 1982. (AGHTM 1984).

Source: Organismes professionnels.

mique sont choisis de sorte que le fluide se vaporise au contact de la source froide (température $T_{2}$ ) en prélevant la chaleur $Q_{2}$ nécessaire à son changement d'état puis se condense au contact de la source chaude (température $T_{1}$ ) avec transfert de la chaleur latente $Q_{1}$ cédée par la condensation. Ce déplacement de calories de la source froide (dans notre cas, l'eau d'une nappe souterraine) vers la source chaude (boucle de chauffage) nécessite, conformément aux principes de la thermodynamique, un apport $W$ d'énergie d'autant plus grand que l'écart $T_{1}-T_{2}$ est élevé. On appelle COP, coefficient de performance du dispositif, le rapport de l'énergie utile $\left(Q_{1}\right.$ dans le cas du chauffage) à l'énergie mécanique fournie $W$ :

$$
\mathrm{COP}=Q_{1} / W=Q_{1} /\left(Q_{1}-Q_{2}\right)
$$

Il en résulte une économie relative d'énergie

$$
\left(Q_{1}-W\right) / Q_{1}=1-1 / \mathrm{COP}
$$

pour un COP de 3, l'économie relative d'énergie est donc $67 \%$ de la chaleur fournie. Du point de vue financier, le bilan économique doit évidemment prendre en compte les coûts propres de chaque énergie (fournie et déplacée).

\section{La pompe à chaleur sur aquifère, avantages, problèmes spécifiques}

Les sources froides les plus exploitées sont l'air extérieur et l'eau de nappe. Dans le premier cas, l'investissement est minime (excepté dispositifs anti-bruit pour les grosses installations), et la ressource infinie sauf les jours froids (au-dessous de $+6^{\circ} \mathrm{C}$ ) lorsque la condensation de l'humidité de l'air sur l'échangeur extérieur accélère la chute du COP déjà amorcée par l'accroissement de l'écart thermique à fournir (mauvais rendement de l'échangeur et dépense d'énergie pour dégivrage périodique), jusqu'à rendre le fonctionnement de la PAC plus onéreux à basse température que la consommation d'une énergie d'appoint.

La pompe à chaleur sur eau de nappe bénéficie au contraire d'une température de source froide quasiment constante; en contrepartie, l'accès aux eaux souterraines nécessite généralement la réalisation d'au moins un forage, dont le coût est très variable suivant la profondeur de la nappe et la nature des terrains à traverser.

Ainsi se développe une demande nouvelle en eaux souterraines, concurrente des exploitations traditionnelles. $\mathrm{Ce}$ besoin nouveau est encore comparativement faible, mais sa rapide croissance, notamment dans plusieurs grandes agglomérations, est une source d'inquiétude. De fait, le système de taxations sur les prélèvements et sur les rejets dans les réseaux d'assainissement, assorties d'une prime à la réinjection, conduit dans bien des cas, à la faisabilité économique de l'installation d'un doublet de forages pour faire fonctionner une PAC sur nappe : l'eau pompée puis refroidie au contact de la PAC, est immédiatement et intégralement réinjectée dans l'aquifère d'origine par un second forage.

Le système du doublet permet le maintien global de la ressource en eau souterraine; dans ces conditions, le développement des PAC sur nappe ne constitue plus une menace pour les autres utilisations de l'eau. Il a toutefois 
l'inconvénient de développer des zones froides d'extension très variable à proximité des forages de réinjection. Celles-ci sont partiellement attirées par le puits d'exhaure du doublet d'origine (refroidissement de la source froide) et partiellement entrainées par l'écoulement de la nappe (gêne pour d'autres utilisateurs).

\section{$\therefore$}

Nous nous efforcerons d'apporter quelques éléments de réponses à différentes questions qui surgissent lorsqu'une telle installation est envisagée :

- Quels sont les éléments à connaitre sur une nappe pour implanter les forages du doublet?

- Où trouver ces informations ?

- Quel sera le coût des ouvrages ?

- Quel débit peut-on atteindre en pompage et en injection?
- Quels sont les risques d'évolution de ces performances dans le temps?

- Peut-on s'assurer contre ces différents risques ?

- Quelle est la vitesse locale de la nappe (renouvellement des calories exploitées)?

- Quel sera le taux de recyclage du front froid, et comment implanter le forage pour réduire ce taux ?

- Comment évolueraient les températures après percée du front froid?

- Quel sera l'impact des réinjections sur les températures du réservoir aquifère?

- Peut-on procéder à une recharge saisonnière de l'énergie des nappes, ou même à un véritable stockage thermique ? - Quelle est la législation française ? Doit-on demander des autorisations avant de réaliser les forages?

- Quelles sont les taxations sur les prélèvements et les rejets?

- Peut-on envisager pour le futur une gestion optimisee hydraulique et thermique des aquifères dans les zones à forte exploitation?

\section{Aspects hydrogéologiques}

\section{Connaissance de la nappe}

Transmissivité de l'aquifère et profondeur du niveau piézométrique sous le sol conditionnent le débit exploitable. La nature des sols à traverser et surtout de l'aquifère ainsi que la profondeur de celui-ci sous le sol conditionnent le coût du forage et de son équipement, notamment en réinjection.

Les exigences sur la qualité des eaux sont bien moindres que pour les alimentations en eau potable, mais le bon fonctionnement de l'échangeur requiert de l'eau non corrosive ni abrasive, ni incrustante et l'absence de certaines bactéries (ferro-oxydantes, sulfato-réductrices) susceptibles de colmater l'ouvrage d'injection et même d'endommager la pompe d'exhaure. On peut, dans certains cas, être contraint d'interposer un échangeur intermédiaire, entre l'eau du forage et la pompe à chaleur, d'équiper le forage d'acier inoxydable et/ou de prévoir des traitements périodiques des eaux.

L'écoulement naturel de la nappe et la proximité du sol conditionnent le renouvellement énergétique local.

\section{Sources d'information}

Ces différents paramètres locaux peuvent être obtenus par des forages de reconnaissance et des essais sur site; mais le coût de ces opérations, très généralement prohibitif, conduit à rechercher des sources d'informations même imprécises afin d'orienter les projets.

Les conditions requises par les installations de faible puissance sont remplies dans la plus grande partie de l'hexagone; les zones favorables aux exploitations à fort débit, sont beaucoup plus restreintes (AGHTM, 1984).

Dans nombre de régions, la productivité est prévisible (fig. 3), par exemple :

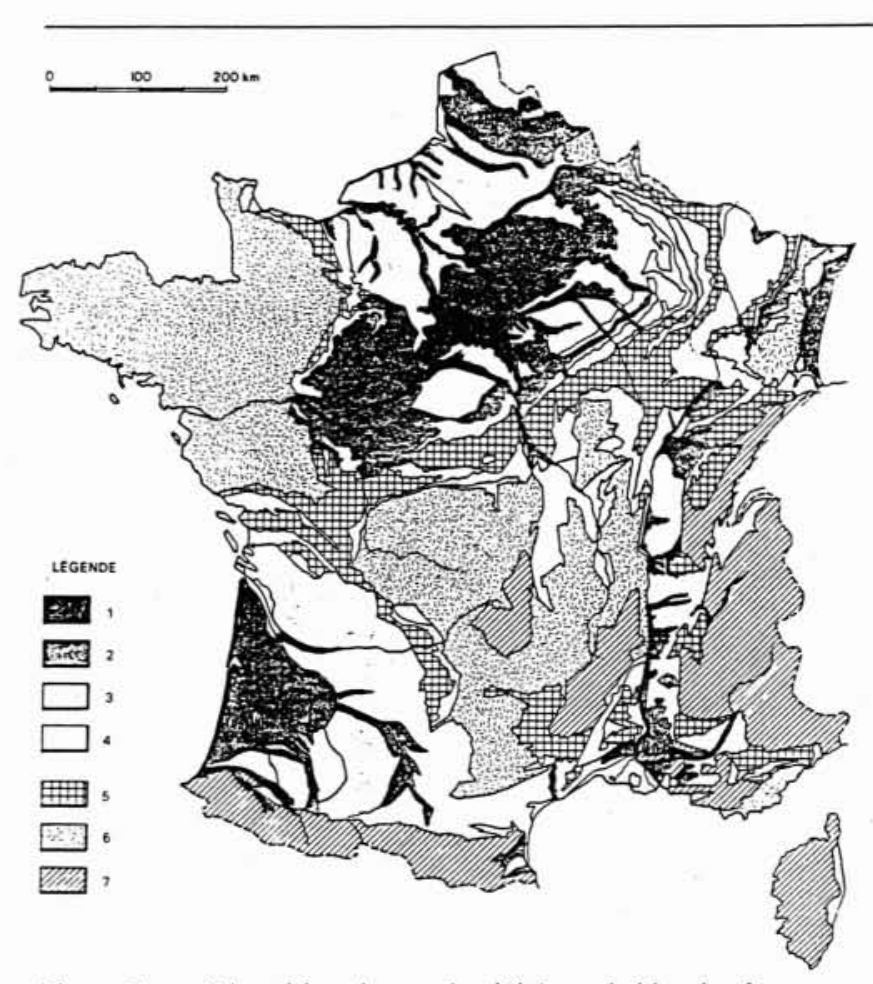

Figure 3. - Répartition des productivités probables des forages d'exploitation d'eau, relatives à la première nappe accessible, en France. (AGHTM, 1984).

- Zones à productivité probable prévisible ( $>50 \%$ des forages) : $1:>100 \mathrm{~m}^{3} / \mathrm{h}-2: 10$ à $100 \mathrm{~m}^{3} / \mathrm{h}-3: 1$ à $10 \mathrm{~m}^{3} / \mathrm{h}-4$ : $<1 \mathrm{~m}^{3} / \mathrm{h}$ (domaine sans nappe d'eau souterraine accessible à faible profondeur, sauf très localement)

- Zone à productivité variée, mal prévisible :

5 : Aquifères calcaires karstiques à productivité très variable et aléatoire $\left(1\right.$ à $\left.>100 \mathrm{~m}^{3} / \mathrm{h}\right)-6$ : Aquifères discontinus de socle cristallin ou schisteux à productivité fréquente de 1 à quelques $\mathrm{m}^{3} / \mathrm{h}$, possible mais plus aléatoire jusqu'à 10 ou plusieurs dizaines de $\mathrm{m}^{3} / \mathrm{h}-7$ : Domaine montagneux non classé. 
- productivité élevée des principales nappes alluviales et des pays crayeux du Bassin Parisien et du Nord; - productivité faible des domaines argileux des bassins sédimentaires;

Elle est aléatoire dans les régions hétérogènes et complexes :

- pays des massifs anciens, généralement faible, localement moyenne;

- pays des plateaux karstiques à productivité extrêmement variable avec des niveaux généralement profonds.

Les critères économiques excluent généralement les aquifères relativement profonds (par exemple Causse, Aquitaine centrale...).

Les caractéristiques de ces aquifères peuvent être estimées à l'aide des cartes géologiques et hydrogéologiques mais cette approche requiert des connaissances spécialisées. Un certain nombre de synthèses régionales ont abouti à une cartographie orientée vers l'utilisateur de pompe à chaleur. les paramètres hydrogéologiques sont remplacés par des données plus élaborées, résultant d'interprétations : c'est ainsi qu'une cartographie réalisée pour la région Ile de France (fig. 4) remplace les transmissivités par les débits exploitables sous $3 \mathrm{~m}$ de rabattement (chute de pression correspondant à $3 \mathrm{~m}$ de hauteur d'eau). Ces cartographies à grande échelle fournissent un ordre de grandeur des paramètres dans les zones homogènes; une présomption de potentialité dans les zones hétérogènes. Elles servent à l'orientation des pré-études de faisabilité. Pour aborder l'étude du projet définitif d'une installation de quelque importance, il sera sage de consulter un hydrogéologue local, par exemple auprès du Service Géologique Régional. La connaissance des aquifères qu'il pourra compléter par une étude de forages voisins éventuels permet souvent une obtention rapide des renseignements recherchés... lors de la préparation du projet, alors qu'il n'est pas rare de voir installer une pompe à chaleur eau-eau dans une habitation préalablement à la réalisation d'un forage... sec.

\section{Coût des forages}

Le coût des ouvrages dépend de :

- la situation géographique (amenée et repli du matériel de forage);

- la nature des terrains à traverser (choix du matériel, et durée de la foration ne sont pas les mêmes dans un granite ou dans des sables et graviers alluvionnaires; tubage éventuel);

- la nature de l'aquifère (équipement en crépine, massif filtrant, traitements mécaniques ou chimiques pour accroître la perméabilité à proximité immédiate de l'ouvrage); - la profondeur de l'aquifère sous le sol (profondeur de l'ouvrage),

- la profondeur du niveau piézométrique sous le sol, la transmissivité de l'aquifère, et le débit à extraire (diamètre de l'ouvrage et choix de la pompe).

Les investissements dans l'installation d'une pompe à chaleur sur nappe étant importants, les forages d'exhaure et de réinjection doivent donc être conçus avec un maximum de soin et tenir compte des conditions d'exploitation difficiles: arrêts et démarrages fréquents peu fa-
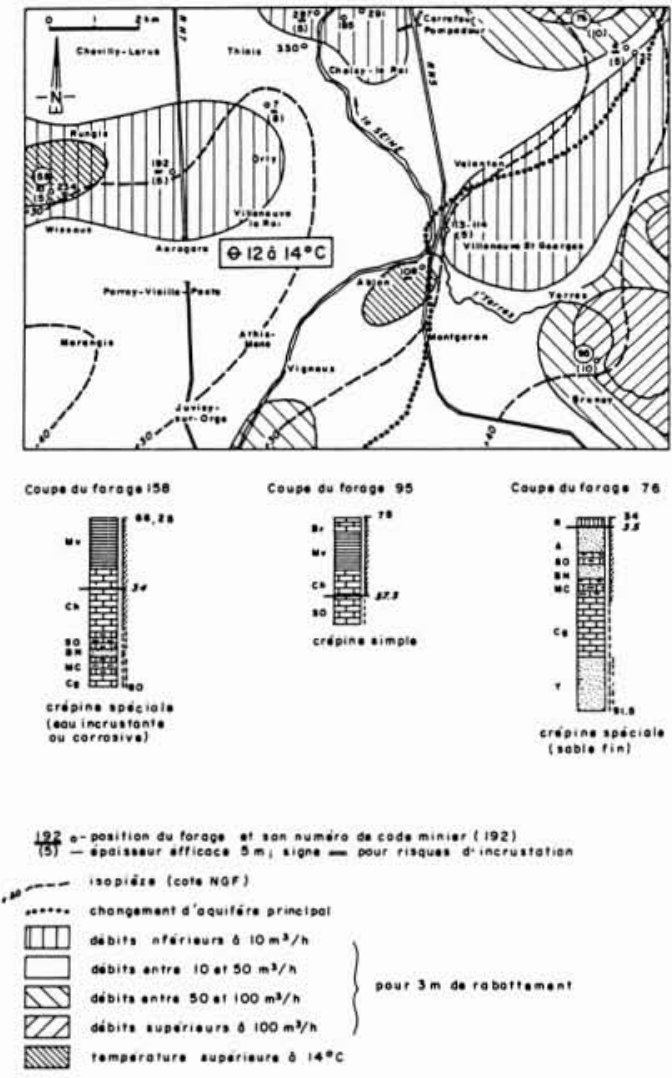

Figure 4. - Extrait d'une carte d'analyse établie pour la Région Ile-de-France (Campinchi, 1982).

vorables à la pérennité des ouvrages. On peut y pallier par un fonctionnement à des débits sensiblement inférieurs aux possibilités maximales des ouvrages, par l'installation d'un variateur de débit sur les pompes, permettant une montée progressive en régime... L'avis d'un homme de l'art sera précieux.

\section{Débit exploitable}

Le débit est généralement plafonné par le rabattement maximal que l'on peut imposer dans le puits d'exhaure et par la surcharge maximale dans le puits d'injection. Le rabattement dans un puits unique (sans recharge à proximité), a été étudié depuis longtemps par les pétroliers et les hydrogéologues. Il est approché par la formule de Jacob, valable à la distance $r$ de l'axe du puits (le rabattement dans le puits correspond en première approche à $r=$ rayon du puits).

$$
s=\frac{Q}{4 \Pi K h} \operatorname{Ln}\left(\frac{2,25 K h}{r^{2} S} t\right)
$$

avec :

$Q$ débit exploité $\left(\mathrm{m}^{3} \mathrm{~s}^{-1}\right)$

$K$ perméabilité $\left(\mathrm{m} \mathrm{s}^{-1}\right)$

$h$ épaisseur en eau (m)

$S$ coefficient d'emmagasinement (sans dimension)

$r$ distance (m)

$t$ durée de l'exploitation en secondes

Ln logarithme naturel 
Cette formule permet d'exprimer le débit $Q$ exploitable en fonction de $s$, rabattement admissible.

La recharge dans le puits d'injection est théoriquement symétrique. En fait, la perméabilité décroît dans de nombreux cas au voisinage immédiat de ce puits (milieux poreux relativement fins, grès argileux...). Une perte de charge supplémentaire doit être vaincue pour faire pénétrer l'eau d'injection, elle peut résulter de différents phénomènes globalement désignés sous le terme de colmatage :

- obturation d'une partie des pores par des bulles de gaz résultant d'une aération des eaux lors de leur transit entre les deux puits, ou d'un simple dégazage au cours de la décompression;

- modification mécanique de l'arrangement des particules lorsque sur un même forage, on passe du pompage à l'injection;

- précipitations géochimiques sous l'effet des variations thermiques de la boucle;

- développement de bactéries.

L'injection ne pose généralement pas de problème dans un horizon calcaire, surtout si l'on a pris la précaution d'améliorer sa perméabilité au voisinage du forage par acidification préalable.

\section{Risque sur les débits, la procédure AQUAPAC}

Dans les aquifères complexes et hétérogènes, inégalement prospectés, subsiste un "risque géologique " d'échec du forage (débit très inférieur aux prévisions). Le maitre d'ouvrage d'une installation de PAC est souvent moins averti et préparé que d'autres exploitants d'eau souterraine à courir ce risque, seul élément d'incertitude dans une opération où le reste est assuré clef en main (AGHTM,
1984). C'est pourquoi la procédure "AQUAPAC " promue par un groupement AFME-BRGM-EDF garantit la ressource en eau souterraine à faible profondeur (jusqu'à $100 \mathrm{~m}$ ), utilisée à des fins énergétiques.

Une double garantie, offerte par AQUAPAC, concerne le risque géologique initial et la pérennité des débits de l'installation sur les 5 premières années. La ressource énergétique effectivement exploitable est évaluée sur la base d'un prélèvement de $5^{\circ} \mathrm{C}$ sur l'eau pompée. Le coût de cette assurance est de l'ordre de $10 \%$ du prix de l'ouvrage pour le risque géologique, et de $3 \%$ pour la garantie de pérennité. Dans le cas où la réinjection est nécessaire, le forage correspondant est inclus dans la garantie.

Les comités régionaux AQUAPAC octroient les garanties au vu des dossiers qui leur sont soumis; ils peuvent demander un complément d'étude (subventionné à $50 \%$ ) si l'instruction des dossiers leur apparaît insuffisante.

\section{Vitesse locale des nappes}

L'impact thermique du rejet froid du doublet sur son propre puits d'exhaure et sur l'aquifère est étroitement lié aux écoulements de la nappe; plus exactement aux flux hydrauliques mesurés par la vitesse de Darcy $\overrightarrow{\mathrm{v}}$, en grandeur et en direction, avec :

$$
\overrightarrow{\mathrm{V}}=K \overrightarrow{\operatorname{grad}} H ; V=|\vec{V}|=K i
$$

avec

$K$ perméabilité

$H$ charge hydraulique

$i$ pente locale de la nappe (module du gradient)

Son évaluation passe par une estimation de la perméabilité et par l'observation de la piézométrie locale.

\section{Comportement thermique du sous-sol}

\section{Transferts thermiques}

L'injection d'eau à une température différente de celle de la formation géologique perméable crée une perturbation thermique qui se propage dans le sens de l'écoulement (convection) mais à une vitesse nettement inférieure à celle de la nappe, une part importante des calories étant captée par la roche en place (effet capacitif). Les vitesses locales résultent de la composition de l'écoulement régional et de l'influence des puits voisins (pompage et injection).

La perturbation thermique est atténuée du fait des échanges conductifs avec les formations voisines (épontes) et de la dispersion au sein de l'aquifère (variabilité spatiale des perméabilités) : un front thermique initialement abrupt est progressivement remplacé au cours de son déplacement dans l'aquifère, par une zone de transition d'extension croissante. L'effet d'échange avec l'éponte supérieure devient vite prépondérant si celle-ci est peu épaisse : la proximité du sol avec sa température imposée par les échanges atmosphériques a tôt fait d'effacer les perturba- tions dans une nappe située à un ou quelques mètres sous le sol.

Du fait des faibles contrastes de température intervenant ici, les gradients de densité et de viscosité de l'eau seront considérés sans conséquence pratique (SAUTY, 1981).

Des modèles mathématiques ont été mis au point qui permettent de simuler les transferts de chaleur dans le sol mais pour nombre d'applications pratiques, des abaques généraux et formules approchées ont été développés qui fournissent des ordres de grandeur suffisants au stade des pré-études, lorsque les valeurs des paramètres du sol (et les conditions d'exploitation) restent relativement imprécises.

\section{Taux de recyclage, baisse de la température d'exhaure}

La proportion $I$ des eaux recyclées peut être calculée en fonction de deux paramètres adimensionnels $\alpha$ et $Q_{R}$ qui 

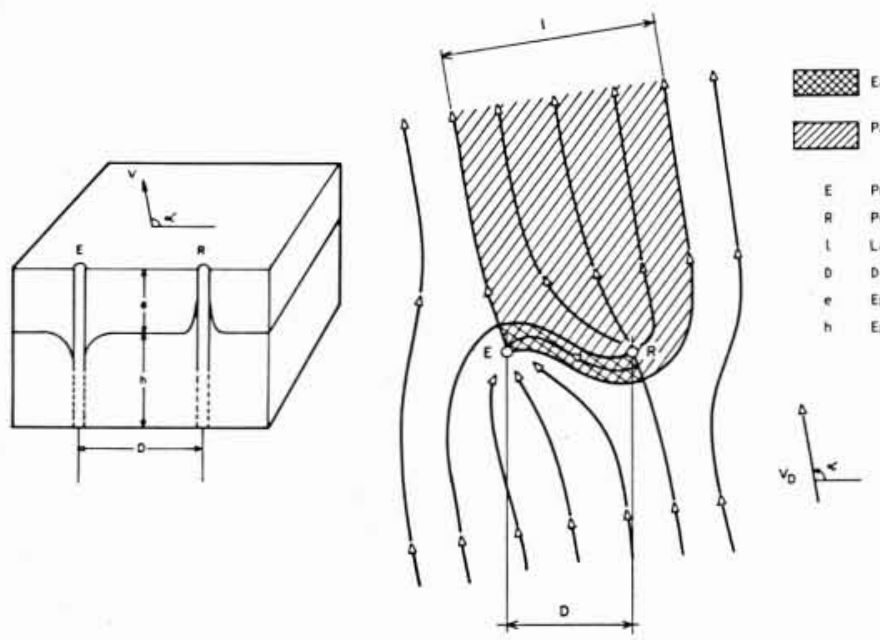

Figure 5. - Exploitation par doublet dans une nappe en écoulement.

a) perspective

b) lignes de courant vues en plan horizontal

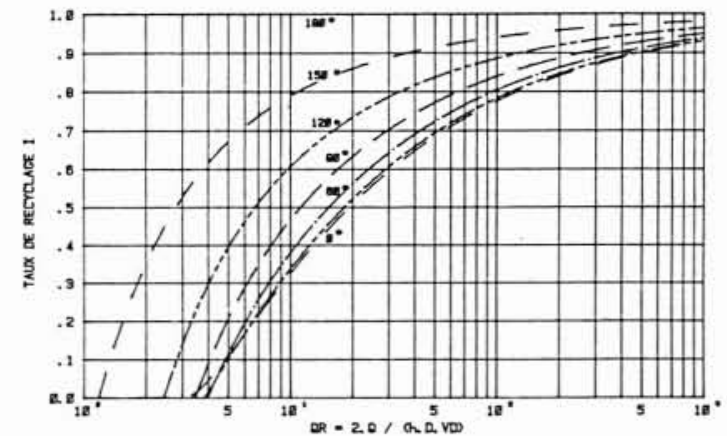

Figure 7. - Doublet parallèle à l'écoulement régional. Injection en aval. Influence du débit du doublet.
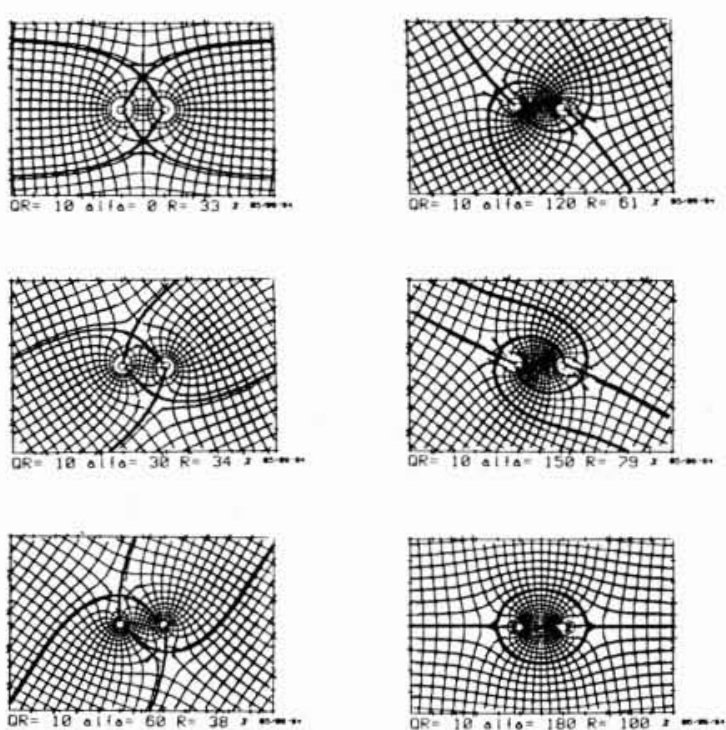

Figure 6. - Influence de l'orientation du doublet sur le réseau d'écoulement et le taux de recyclage.

définissent entièrement le réseau d'écoulement :

$\alpha=$ angle $(E \vec{R}, \vec{V})$ est l'angle que fait la direction de l'écoulement avec l'axe du doublet, orienté du puits d'exhaure vers le puits de réinjection;

$Q_{R}=2 Q /(h D V)$ caractérise le rapport du débit du doublet au débit de la nappe (notations : fig. 5).

La figure 6 met en évidence l'influence de l'angle $\alpha$ d'implantation du doublet sur le réseau d'écoulement. Dans la pratique, afin de réduire le taux de recyclage, on aura intérêt à orienter le doublet suivant l'axe de l'écoulement naturel avec pompage en amont et injection en aval $(\alpha=0)$. La figure 7 montre justement l'influence du débit dans cette hypothèse.

Un abaque (fig. 8) a été construit pour obtenir directement I sans être contraint de recourir à une simulation par modèle mathématique.

Une exploitation du doublet avec prélèvement d'un écart thermique constant $\Delta \theta$, fait évoluer progressivement la température des eaux pompées sur le puits d'exhaure, de la valeur initiale $\theta_{0}$ à la valeur $\theta_{S}$ à stabilisation, telle que :

$$
\theta_{S}=\theta_{0}+\Delta \theta I /(1-I)
$$

\section{Impact thermique : développement et stabilisation du panache}

Du fait de l'écoulement régional, la fraction $(1-I) Q$ du débit réinjecté est entraînée par advection hors de portée du puits d'exhaure. La largeur du panache croît progressivement jusqu'à ce que l'influence régionale l'emporte sur celle des deux puits; les vitesses à l'intérieur du panache deviennent alors parallèles à la direction de l'écoulement régional (fig. 5).

La largeur $l$ du panache croît vers la valeur :

$l=Q(1-I) /(V h) ;$ la perturbation thermique se propage à la vitesse $V_{t h}$ :

$$
V_{t h}=\left(\gamma_{F} / \gamma_{A}\right) V
$$

$\left(V_{t h} \sim 1,7 \mathrm{~V}\right.$ en moyenne pour une nappe alluviale)

C'est ainsi que pour un débit de $10 \mathrm{~m}^{3} / \mathrm{h}$ (dont $30 \%$ recyclés) en moyenne sur la période de chauffe, extraits d'une nappe de $5 \mathrm{~m}$ d'épaisseur, elle-même siège d'un écoulement régional de $40 \mathrm{~m} / \mathrm{an}$, la largeur du panache est de l'ordre de $300 \mathrm{~m}$.

Théoriquement, l'extension du panache se développe indéfiniment vers l'aval avec la durée de l'exploitation, le 

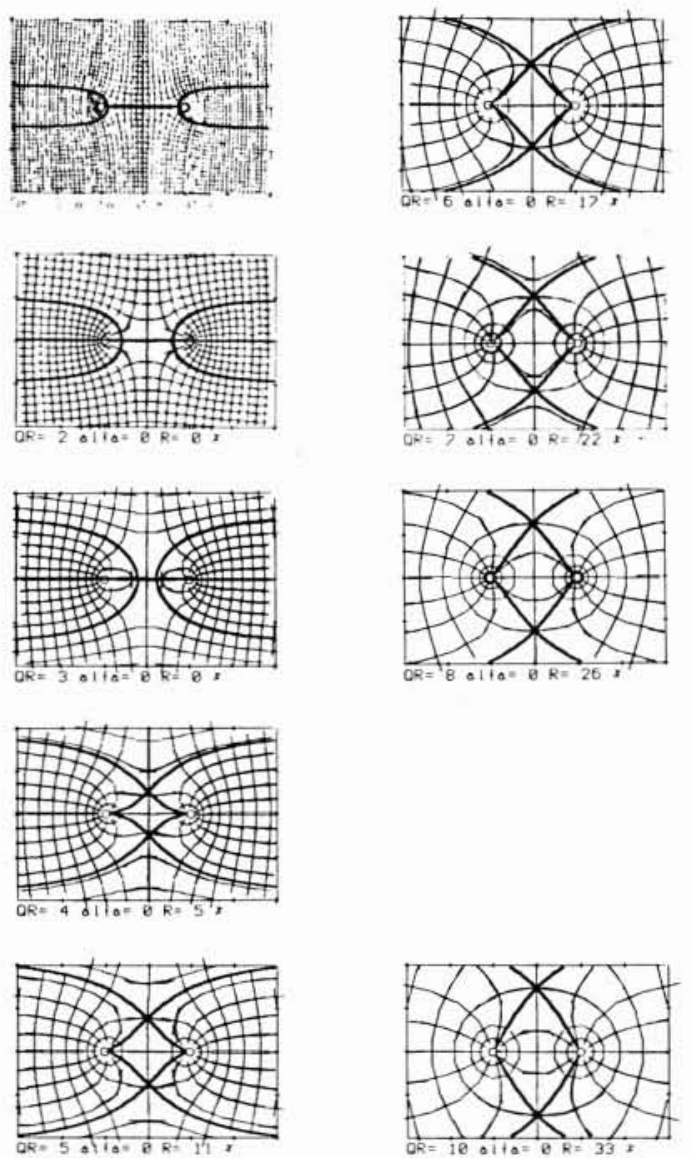

Figure 8. - Taux de recyclage en fonction de $Q_{d}$ et de alpha.

flux géothermique étant impuissant - du moins à l'échelle de temps humaine - pour restituer à l'aquifère sa température naturelle. En pratique, cet impact n'est cependant sensible que sur une longueur limitée, du fait de la diffusion de la perturbation par conduction thermique dans le milieu environnant. La proximité du sol, dont la température est imposée par les conditions de surface, limite rapidement la progression du panache dans les aquifères à très faible profondeur ( 1 à quelques mètres).

Des formulations permettent de calculer l'évolution initiale ainsi que la stabilisation finale (Ausseur, Sauty, 1982).

Une formule approchée a été proposée (M. Grima, 1984) qui permet de couvrir avec une bonne approximation l'ensemble de l'évolution (notations en annexe) :

$$
\begin{aligned}
\Delta \theta_{R}=\operatorname{Min}\left\{\operatorname{erfc} \sqrt{\Lambda\left(t_{R}-1\right)},\right. & \\
& \left.\quad \exp (-1 / \Lambda E) \operatorname{erfc}\left[\frac{1}{2} \sqrt{\Lambda\left(t_{R}-1\right)}\right]\right\}
\end{aligned}
$$

A titre d'exemple, on a évalué l'amplitude de la perturbation thermique à différentes distances du point de rejet, en fonction de l'épaisseur de l'éponte supérieure, comprise entre 1 et $100 \mathrm{~m}$, pour $V=50 \mathrm{~m} / \mathrm{an}, h=10 \mathrm{~m}$, dans l'hypothèse d'une éponte supérieure humide. On constate le fort contrôle que l'épaisseur de cette éponte

\begin{tabular}{|c|c|c|c|c|c|c|c|}
\hline \multicolumn{8}{|c|}{$\begin{array}{l}\text { TABLEAU I. - Écarts thermiques } \\
\text { à stabilisation pour différentes épaisseurs } \\
\text { d'éponte et distances au point de rejet; } \\
\text { les écarts sont exprimés en fraction } \\
\text { de l'écart thermique }\left(\Delta \theta=\theta_{i}-\theta_{0}\right) \text { à l'injection } \\
\text { (d'après Ausseur, Sauty, 1982) }\end{array}$} \\
\hline$x(m) \quad e(m)$ & 1 & 2 & 5 & 10 & 20 & 50 & 100 \\
\hline 10 & $69 \%$ & $83 \%$ & $93 \%$ & $96 \%$ & $98 \%$ & $99 \%$ & $100 \%$ \\
\hline 20 & $47 \%$ & $69 \%$ & $86 \%$ & $93 \%$ & $96 \%$ & $98 \%$ & $99 \%$ \\
\hline 50 & $15 \%$ & $39 \%$ & $69 \%$ & $83 \%$ & $91 \%$ & $96 \%$ & $98 \%$ \\
\hline 100 & $2 \%$ & $15 \%$ & $47 \%$ & $69 \%$ & $83 \%$ & $93 \%$ & $96 \%$ \\
\hline 200 & $0 \%$ & $2 \%$ & $22 \%$ & $47 \%$ & $69 \%$ & $86 \%$ & $93 \%$ \\
\hline 500 & $0 \%$ & $0 \%$ & $2 \%$ & $15 \%$ & $39 \%$ & $69 \%$ & $83 \%$ \\
\hline 1000 & $0 \%$ & $0 \%$ & $0 \%$ & $2 \%$ & $15 \%$ & $47 \%$ & $69 \%$ \\
\hline 2000 & $0 \%$ & $0 \%$ & $0 \%$ & $0 \%$ & $2 \%$ & $22 \%$ & $47 \%$ \\
\hline 5000 & $0 \%$ & $0 \%$ & $0 \%$ & $0 \%$ & $0 \%$ & $2 \%$ & $15 \%$ \\
\hline 10000 & $0 \%$ & $0 \%$ & $0 \%$ & $0 \%$ & $0 \%$ & $0 \%$ & $2 \%$ \\
\hline $\begin{array}{l}\text { arrivée au sol } \\
\text { de la pertur- } \\
\text { bation ( } 1 \%)\end{array}$ & $1 \mathrm{jr}$ & $4 \mathrm{jrs}$ & $22 \mathrm{jrs}$ & $90 \mathrm{jrs}$ & 1 an & 6 ans & 24 ans \\
\hline
\end{tabular}
exerce sur l'extension de la perturbation ( tableau I).

\section{Aspects législatifs Gestion de la ressource}

\section{Les besoins en eau des PAC sur nappe}

L'estimation par le Comité français d'électrothermie, de 50000 équivalents-logements chauffés par PAC/eau en 1986 équivaut à un prélèvement brut de 150 millions de $\mathrm{m}^{3} / \mathrm{an}$ et il n'est pas absurde de présumer que ces consommations se chiffreront par plusieurs centaines de millions de $\mathrm{m}^{3} /$ an vers la fin du siècle (AGHTM, 1984).

Les grandes agglomérations pourvues en eaux souterraines d'accès facile concentrent une part importante des installations : Strasbourg, Lyon, Paris. A Strasbourg, dans la nappe du Rhin dont le débit naturel moyen a été estimé à $160 \mathrm{Mm}^{3} / \mathrm{an}$, les débits pompés annuels représentaient $120 \mathrm{Mm}^{3} / \mathrm{an}$ en 1980 dont plus de $20 \%$ à des fins thermiques (Mehreb, 1984). Dans le Département du Rhône, $1650 \mathrm{~m}^{3} / \mathrm{h}$ sont exploités par pompe à chaleur, dont 1150 au sein de la communauté urbaine (rapport PACRA : Association Rhonalp des pompes à chaleur, état en janvier 1984).

Même si la réinjection devenait la règle et éliminait le problème quantitatif de la ressource en eau, des contentieux dus aux interférences thermiques seront inévitables dans un avenir proche, du moins dans les zones à forte concentration. peut-on concevoir une gestion des ressources sous le double aspect hydraulique et thermique? Quelle est la législation actuelle? 


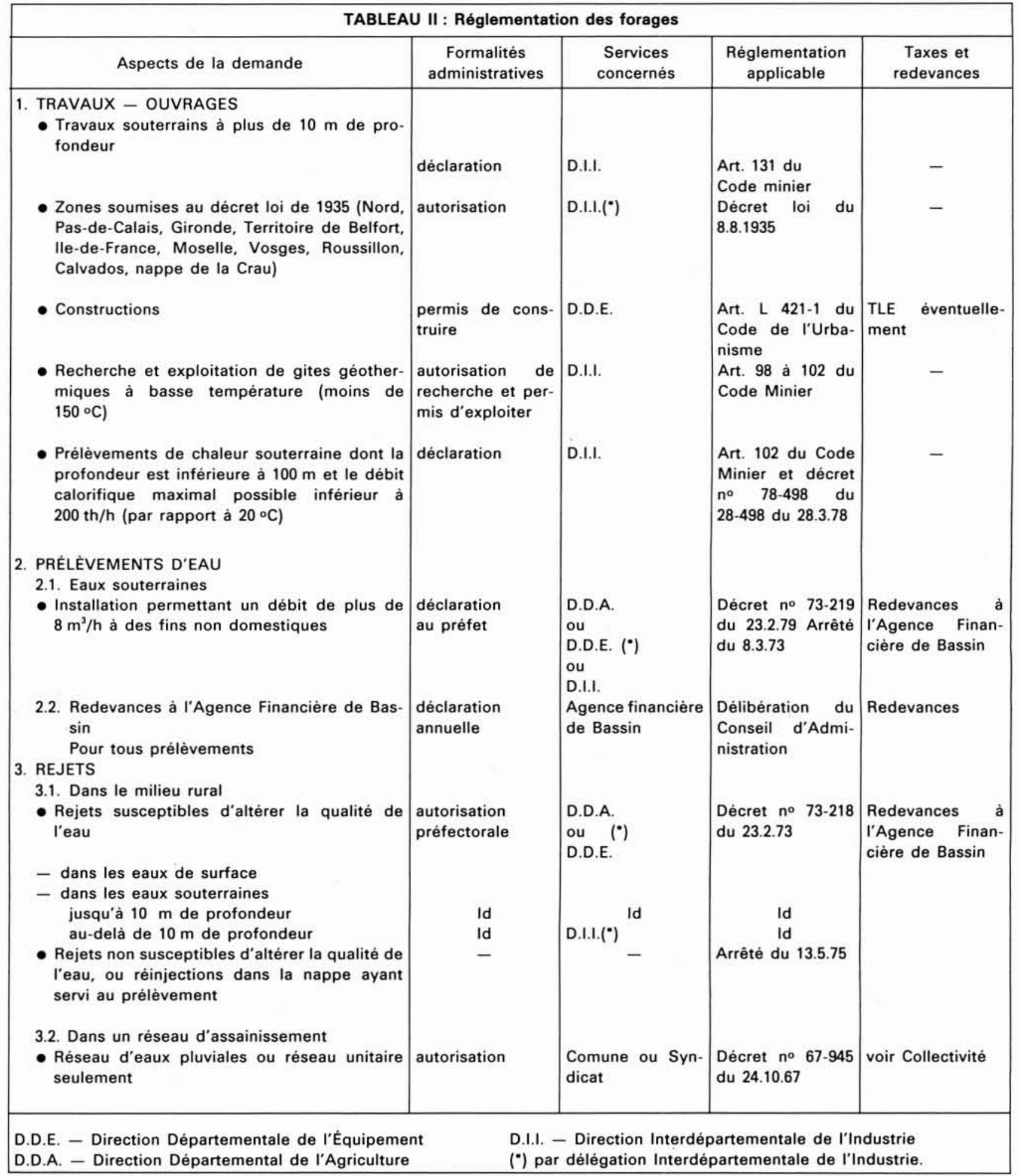

\section{La réglementation française (d'après AGHTM, 1984)}

Seules, les exploitations profondes (100 m et plus), sont assujetties au code minier relatif aux gites géothermiques à basse température (ainsi que les forts débits sur des eaux moins profondes à plus de $20^{\circ} \mathrm{C}$, cas particulièrement rare): demande d'autorisation de recherche et permis d'exploitation.

Les forages moins profonds ressortissent du régime général des prélèvements d'eau souterraine. La réglemen- tation destinée à prévenir les conflits d'usage, n'est pas unique, elle dépend des débits prélevés, des profondeurs, et des régimes. La réglementation des rejets en tant qu'actes susceptibles d'altérer la qualité de l'eau souterraine, est par contre plus générale.

Le tableau II récapitule les formalités à remplir pour réaliser un forage d'eau et l'exploiter.

Le tableau III établi en 1982 pour les Hauts de Seine compare les coûts entraînés par les taxations attachées à différents types de rejet (Campinchi, 1982). 


\begin{tabular}{|c|c|c|c|c|c|c|c|}
\hline \multicolumn{8}{|c|}{$\begin{array}{l}\text { TABLEAU III : Taxation d'un doublet en fonction } \\
\text { du mode de rejet (Campinchi, 1982) }\end{array}$} \\
\hline \multirow{2}{*}{ Cas rencontrés } & \multicolumn{4}{|c|}{ Taxes } & \multirow{3}{*}{$\begin{array}{l}\begin{array}{c}\text { Primes de } \\
\text { I'Agence }\end{array} \\
\mathrm{Pr}=0,0948 \\
\mathrm{~F} / \mathrm{m}^{3}\end{array}$} & \multirow{2}{*}{\multicolumn{2}{|c|}{$\begin{array}{c}\text { Exemple pour } \\
500000 \mathrm{~m}^{3} / \mathrm{an} \\
\text { prélevés et } \\
\text { réinjectés } \\
\left(57 \mathrm{~m}^{3} / \mathrm{h}\right)\end{array}$}} \\
\hline & Agence & Région & $\begin{array}{l}\text { Dépar- } \\
\text { tement }\end{array}$ & Commune & & & \\
\hline 1) Rejet en aquifère souterrain & \multicolumn{4}{|c|}{$\mathrm{Tp}=0,1019 \mathrm{~F} / \mathrm{m}^{3}$} & & $\begin{array}{r}50950 \mathrm{~F} \\
-47400 \mathrm{~F}\end{array}$ & $3550 F$ \\
\hline $\begin{array}{l}\text { 2) Rejet dans réseau d'eau de } \\
\text { surface (idem pour épan- } \\
\text { dage sur le sol) }\end{array}$ & $\begin{array}{l}\mathrm{Tp} \\
\mathrm{Tr}=0\end{array}$ & & & & & & $50950 \mathrm{~F}$ \\
\hline $\begin{array}{l}\text { 3) Rejet dans réseau séparatif } \\
\text { pluvial }\end{array}$ & $T p$ & & & $T r p$ & & $\begin{array}{l}50950 F \\
+ \text { variable }\end{array}$ & $>50950 \mathrm{~F}$ \\
\hline $\begin{array}{l}\text { 4) Rejet dans réseau d'assai- } \\
\text { nissement ou dans réseau } \\
\text { unitaire }\end{array}$ & $\begin{array}{l}\text { Tp } \\
\text { Contre-valeur } \\
\text { au-delà des } \\
6000 \text { premiers } \\
\mathrm{m}^{3} \text { prélevés } \\
0,27 \mathrm{~F} / \mathrm{m}^{3}\end{array}$ & $0,47 \mathrm{~F} / \mathrm{m}^{3}$ & $0,32 \mathrm{~F} / \mathrm{m}^{3}$ & $\begin{array}{l}0,07 \text { à } \\
0,40 \mathrm{~F} / \mathrm{m}^{3} \\
\text { moy. : } \\
0,23 \mathrm{~F} / \mathrm{m}^{3}\end{array}$ & & $\begin{array}{l}133380 \mathrm{~F} \\
210000 \mathrm{~F} \\
160000 \mathrm{~F} \\
115000 \mathrm{~F}\end{array}$ & $669330 \mathrm{~F}$ \\
\hline \multicolumn{8}{|c|}{$\begin{array}{l}T p=\text { Taxe de prélèvement. } \mathrm{Tr}=\text { Taxe pour rejet. } \mathrm{Trp}=\text { Taxe pour rejet dans un réseau pluvial séparatif. } \mathrm{Pr}=\text { Prime pour rejet } \\
\text { dans aquifère }=9,3 \% \text { de } \mathrm{Tp} .\end{array}$} \\
\hline
\end{tabular}

Soulignons enfin que l'eau souterraine appartient à celui qui en a l'usage une fois l'eau extraite : aucun texte ne protège un exploitant contre le fait qu'un pompage voisin peut assécher son captage (sauf malveillance intentionnelle).

\section{Un modèle de gestion hydrothermique}

Le développement singulièrement dense des doublets thermiques dans le tissu urbain Strasbourgeois a conduit à l'élaboration d'un programme pluriannuel comportant (Mehreb et Babot, 1983) :

- la collecte des informations concernant les prélèvements d'eau à des fins diverses;

- l'acquisition des données sur les installations thermiques : prélèvements, puissances calorifiques, rejets;

- un approfondissement des connaissances sur la nappe d'Alsace sous l'agglomération de Strasbourg : géométrie, caractéristiques hydrauliques, alimentations, infiltration de la pluie, échanges avec les cours d'eau;

- suivi des températures dans les forages mais aussi les caves et garages en sous-sol;

- mise au point d'un modèle mathématique (fig. 9) simulant les écoulements et les transferts de chaleur dans la nappe (Mehreb, 1984).

L'utilisation de cet outil de gestion, mis à disposition des décideurs pourra servir à :

- évaluer les bilans hydrauliques thermiques et économiques découlant d'hypothèses d'exploitation variées;

- préserver les diverses utilisations de la nappe;

- optimiser l'exploitation thermique de ces ressources.

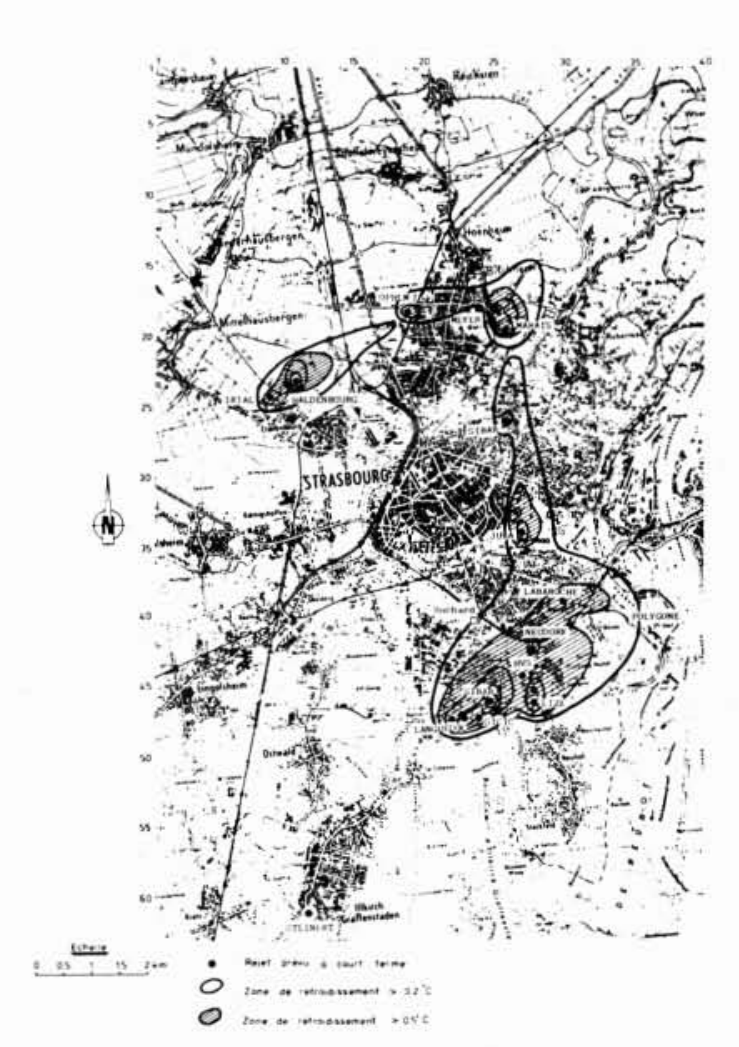

Figure 9. - Utilisation de la nappe phréatique d'Alsace au niveau de Strasbourg. Modèle hydrothermique. 


\section{Conclusions}

L'installation d'une pompe à chaleur sur doublet requiert un minimum de connaissances sur l'aquifère à exploiter.

Des moyens existent pour prévoir le comportement hydraulique et thermique ainsi que leur impact. Dans les zones à forte densité d'exploitation, la mise en œuvre de modèles de simulation par les collectivités est possible pour définir une gestion optimisée de la ressource. L'efficacité en est toutefois suspendue à la mise en place d'une réglementation appropriée.

\section{Références bibliographiques}

L'eau et les pompes à chaleur - Groupe de travail sur le thème 2 : "L'eau souterraine et les pompes à chaleur ", rapporteur J. MARGAT - Groupe de travail sur le thème $4:$ : Les pompes à chaleur et la législation", rapporteurs M. LAFON et M. PORTNOI $-64^{\circ}$ congrès de l'AGHTM (Association générale des Hygiénistes et Techniciens Municipaux), Clermont-Ferrand, 4-8 juin 1984

AQUAPAC. - Plaquette d'information, éditée par le groupement Aquapac (Agence Française pour la Maîtrise de l'Energie, Électricité de France et le Bureau de Recherches Géologiques et Minières), septembre 1984 .

Ausseur (J.Y.), SAUTY (J.P.). - Exploitation de doublets de forages pour le chauffage ou la climatisation avec pompes à chaleur - Fonctionnement et impact sur l'aquifère. - In : Colloque national en hommage à Gilbert CASTANY à l'occasion de son jubilé scientifique - Orléans, 16-17 nov. 1982, BRGM Documents $\mathrm{n}^{\circ} 45$, p. $33-45$.

Ausseur (J.Y.), CAMPINCHI (J.), SAUTY (J.P.). - Pompes à chaleur sur nappe et stockage en aquifère - Annales des Mines, mai-juin 1983, p. 127-144.

BERNIER (J.). - La pompe de chaleur - Mode d'emploi - Tome I - De la théorie à la pratique - PYC édition 1979.

BERNIER (J.). - La pompe de chaleur - Mode d'emploi - Tome II - Applications pratiques - PYC - édition 1981.

CAMPINCHI (J.). - Aptitudes des nappes d'eaux souterraines entre 0 et $100 \mathrm{~m}$ de profondeur en région Ile-de-France, pour alimenter des pompes à chaleur eau/eau - Aspects hydrogéologique, économique, administratif. - In : Colloque national en hommage à Gilbert CASTANY à l'occasion de son jubilé scientifique - Orléans, 16-17 novembre 1982, BRGM Documents $\mathrm{n}^{\circ} 45$, p. $183-192$.

COLLIN (J.J.), JAUDIN (F.), LE NIR (M.). - Pompes à chaleur sur nappe aquifère - L'usage des aquifères - L'information du public face aux aspects spécifiques et aux facteurs limitant cette nouvelle forme d'usage d'énergie. - In: Colloque national en hommage à Gilbert CASTANY à l'occasion de son jubilé scientifique. Orléans, 16-17 nov. 1982 - BRGM Documents $n^{\circ} 45$, p. 201-210.

DUMinil. - Préface au tome I de l'ouvrage de J. Bernier : La pompe à chaleur, 1979.

GRIMA (M.). - Modélisation des bilans thermiques des aquifères à surface libre - Influence de la zone non saturée et l'étude des panaches de rejet de pompe à chaleur. Thèse de $3^{\circ}$ cycle Grenoble, janvier 1984 .

MeHReB (M.). - Modèle de gestion des échanges hydrothermiques dans les nappes souterraines: application à la région de Strasbourg. Thèse soutenue le 25 mai 1984, Université LouisPasteur - Strasbourg, Institut de Mécanique des Fluides.

SAUTY (J.P.). - Du comportement thermique des réservoirs aquifères exploités pour le stockage d'eau chaude ou la géothermie basse enthalpie. - Thèse d'Etat ès Sc. Phys., Grenoble, juin 1981 - BRGM Documents n 33.
La solubilité de la silice diminuant avec la température, des risques de dépôt et donc de colmatage existent, en particulier au niveau du puits le plus froid; le puits de réinjection. Suite à la question de $M$. GRJEBINE portant sur les techniques mises en œuvre pour éviter ces dépôts, $M$. SAUTY fit remarquer qu'en fait ces problèmes sont d'un ordre mineur dans le cas des pompes à chaleur sur doublet car les rejets sont à 5 ou $60^{\circ}$. Des colmatages d'autres types peuvent intervenir (bactéries...) mais leurs effets sur la perméabilité du réservoir restent faibles. Le comportement peut être très différent quand le $\Delta t$ est plus important (stockage de chaleur sur nappe) ou qu'il y a réinjection d'eau plus chaude (dépôts calcaires). Cf. discussion des exposés suivants. 